\title{
Modeling of Liquid Droplet Impingement onto Ti-6Al-4V Substrate
}

\author{
Mason Marzbali, Ali Dolatabadi \\ Department of Mechanical, Industrial and Aerospace Engineering, Concordia University \\ 1455 De Maisonneuve Blvd. W., Montreal, QC, H3G 1M8, Canada \\ mason.marzbali@concordia.ca; ali.dolatabadi@concordia.ca
}

\begin{abstract}
Droplet impingement is of great interest to power generation and aerospace industries due to the accrued cost of maintenance in steam and gas turbines. The repetitive impacts of liquid droplets onto rotor blades, at high relative velocities, result in the blade erosion, which is known as Liquid Impingement Erosion (LIE). In this regard, it is crucial to understand the hydrodynamics of the impact in order to identify the consequent solid response before addressing the LIE problem. To that end, modeling the impact of liquid droplets onto the blade surface is the main objective of the present work. A novel model for Fluid-Solid Interaction is developed that couples the gas-liquid interfacial model with the structural solver using one-way and two-way coupling algorithms. Furthermore, the effect of the solid elasticity on the generated pressure build-up in the liquid and the resulting stress in the solid are investigated.
\end{abstract}

Keywords: Fluid-solid interaction, liquid impingement erosion, droplet impact, CFD, VOF, FEM.

\section{Introduction}

It is a common practice in power generation industries to inject atomized water into gas turbine engines in order to boost the engine power at high ambient temperatures. The heat removal from the air via vaporization of water droplets, known as evaporative cooling, increases the engine output significantly. The main drawback of this cooling method is the substantial reduction of blade life in compressor stages due to Liquid Impingement Erosion (LIE), caused by high frequency droplet impacts. Since the mass flow rate of injected water, and thus the number of sprayed droplets are significant, large droplets are formed from coalescence of smaller droplets on static components. Further downstream, these large droplets, carried with the air flow, impact the rotary parts in the compressor with high relative velocities due to the high linear velocity of the rotating blade, particularly at the blade tip. A similar problem is found in steam turbines due to water condensation and in aero-engines because of small water particles in the air. The consecutive impacts of water droplets at such speeds change the surface roughness on the blades and initiate the surface erosion. Hence, finding new methods to reduce the blade erosion caused by LIE is of high importance.

LIE is an interdisciplinary phenomenon as it involves both fluid dynamics and solid mechanics. Predicting the potential damage to the solid material requires capturing the flow characteristics in the liquid counterpart accurately. Once the fluid flow and solid stress are resolved, the erosion caused by the liquid impact can be determined using fatigue analysis. In 1927 , Honegger [1] shed light on the mechanisms of LIE with a series of experiments. He argued that no erosion is observed prior to the formation of roughness on the surface. After the incubation stage, the erosion rate grows rapidly as the droplets penetrate the uneven surfaces due to the high impingement pressure. The loading and unloading of the pressure force produced by the impact lead to the development of craters on the surface and the material loss occurs at these craters via ductile rupturing according to Bargmann [2]. Once the spatial time history of the pressure field in the liquid and the stress field in solid are obtained from a single impact, the total damage can be predicted by carrying out the fatigue analysis on the solid as explained by Springer [3].

The spreading of a droplet at low impingement velocities has been studied in details by Fukai et al. [4], Chandra and Avedisian [5], Pasandideh-Fard et al. [6] and more recently by Roisman et al. [7]. The entrapment of an air bubble underneath the droplet has been simulated by Mehdi-Nejad et al. [8]. Moreover, the formation of fingers upon impact of water drops was experimentally investigated by Mehdizadeh et al. [9]. Blake and De Conick [10] have extended the molecular-kinetic theory of dynamic wetting by considering the effect of Fluid-Solid Interaction (FSI). It is believed that the first FSI model with one-way coupling was proposed by Honegger [1], in which, a liquid jet impacts a solid wall creating a constant pressure, commonly known as the water hammer pressure. After him, Cook [11] and Engel [12] reported the 1-D steady-state solution 
for the water hammer pressure. Blowers [13] proposed another pressure model to obtain the stress field in the rigid solids, which was decoupled from the pressure field in the liquid.

In addition to analytical methods, numerical simulations have been used to model droplet impact on solid substrates at high velocities. For instance, Adler and Mihora [14] utilized Finite Element Method to study water droplet impact onto a solid substrate at a high velocity. Moreover, several detailed analyses were performed to simulate high speed impact of droplets on rigid solids such as Haller et al. [15] and Huang et al. [16]; however, they only focused on the fluid dynamics of the phenomenon and did not study the stress field in the solid, which is critical for liquid erosion problem. More recently, Li et al. [17] numerically studied the Liquid Droplet Impingement (LDI) onto rigid solids and proposed a 1-D fully coupled FSI model for early stages of impact before the formation of lateral jets and droplet breakup on the surface. The 1-D numerical model developed by Li et al. [17] was further extended to a 2-D model by Zhou et al. [18].

Analytical analysis of high speed droplet impact in 1-D and 2-D in addition to the numerical modeling with a 2-D domain are reported in the open literature. Although, these models have various limitations and shortcomings, they provide valuable insights to the physics behind droplet impingement. 1-D and 2-D models can only represent liquid columns and cylindrical liquid jets, respectively, and not a spherical droplet. Therefore, the main objective of the present work is to implement a 2-D axisymmetric, two-way coupled FSI solver to model the impingement of incompressible spherical liquid droplets onto rigid and elastic substrates. The compressibility effect was addressed in [19] and [20].

In order to model the interfacial flow in the fluid region which contains liquid and gas phases, Volume of Fluid (VOF) method is utilized. The droplet deformation is precisely captured upon impact with impingement velocity of 100 $\mathrm{m} / \mathrm{s}$. In addition, the stress field in the solid substrate is modeled with Finite Element Method (FEM). The coupling between fluid and solid domains is achieved by imposing the stress continuity and no-slip velocity condition on the fluid-solid interface. The transient pressure history in the fluid domain and the stress field in the solid domain are obtained simultaneously by solving the coupled fluid and solid equations with a two-way coupling approach.

\section{Methodology}

\subsection{Governing equations}

Navier-Stokes equations are solved for two incompressible, isothermal and immiscible fluids. The conservation of mass and momentum equations in their transient and incompressible forms are as follows,

$$
\begin{gathered}
\nabla \cdot V_{f}=0 \\
\rho_{f} \frac{D V_{f}}{D t}=\nabla \cdot \sigma_{f}+\rho_{f} F_{b}
\end{gathered}
$$

where $V_{f}$ is the fluid velocity vector, $\rho_{f}$ is the fluid density, $F_{b}$ is body forces acting on the fluid. $\sigma_{f}$ is the Cauchy stress tensor for a Newtonian fluid, including pressure and viscous terms, defined as,

$$
\sigma_{f}=-p_{f} I+\mu_{f}\left(\nabla V_{f}+\nabla V_{f}^{T}\right)
$$

where $p_{f}$ is the fluid pressure, $I$ is the 3 by 3 identity matrix and $\mu_{f}$ is the fluid dynamic viscosity. A single momentum equation is solved for both gas and liquid phases and fluid properties are calculated for the mixture. The fluid flow is assumed to be laminar; hence, no turbulence model is employed in the solver. The energy equation is not considered in the incompressible model since the temperature variation and the heat conduction during the impact are negligible according to Li et al. [21]. The surface tension force is treated as a pressure gradient across the liquid-gas interface and is calculated per unit volume based on the Continuum Surface Force (CSF) model proposed by Brackbill et al. [22]. The VOF model developed by Hirt and Nichols [23] is employed to resolve the droplet interface. In VOF method, a scalar field is defined for volume fraction of liquid phase, $\alpha$, and its value depends on the fraction of the cell volume occupied by this phase indicated by, 


$$
\left\{\begin{array}{cc}
\alpha=0 & \text { Gas phase } \\
0<\alpha<1 & \text { Gas - liquid interface } \\
\alpha=1 & \text { Liquid phase }
\end{array}\right.
$$

where the values between zero and one denote the interface between gas and liquid phases. Since the volume fraction represents the volume occupied by the liquid, it should be advected by the flow field at each time step. The following equation governs the liquid advection,

$$
\frac{\partial \alpha}{\partial t}+\nabla \cdot\left(\alpha V_{f}\right)=0
$$

Following the advection, the interface is reconstructed using the Piecewise Linear Interface Calculation (PLIC) proposed by Youngs [24].

The structural equation to be solved for an elastic and deformable solid is as follows,

$$
\rho_{s} \frac{D V_{s}}{D t}=\nabla \cdot \sigma_{s}+\rho_{s} g
$$

where $\rho_{s}$ is the solid density, $V_{s}$ is the solid velocity, equal to $V_{s}=\frac{\partial U_{s}}{\partial t}$, and $U_{s}$ is the solid displacement. $g$ is gravitational acceleration and $\sigma_{\mathrm{s}}$ is Cauchy stress tensor for the solid described below,

$$
\sigma_{s}=\frac{1}{J} F\left[\lambda_{s}(\operatorname{tr}(S)) I+2 \mu_{S} S\right] F^{T}
$$

where $J$ is determinant of $F$ and $F$ is the deformation gradient tensor defined as,

$$
F=I+\nabla U_{s}
$$

$S$ is obtained from St. Venant-Kirchhoff law,

$$
S=\frac{1}{2}\left(F^{T} F-I\right)
$$

$\lambda_{s}$ and $\mu_{s}$ are Lamé coefficients defined below,

$$
\begin{gathered}
\lambda_{s}=\frac{v_{s} E}{\left(1+v_{s}\right)\left(1-2 v_{s}\right)} \\
\mu_{\mathrm{s}}=\frac{E}{2\left(1+v_{s}\right)}
\end{gathered}
$$

where $v_{s}$ and $E$ are Poisson ratio and Young's modulus of the solid material, respectively.

In FSI model, the spatial distribution of variables, e.g. stress tensor, needs to be communicated through the interface that is in common between the fluid and solid domains. In addition, the mesh displacement has to be calculated at each time step to determine if there is any deformation in the interface. The two-way coupling approach requires the fluid equations to be solved followed by the structural equation. If internal convergence is achieved for the solid displacement, the calculations proceed to the next time step. If the solid convergence check does not pass, the displacement is under-relaxed and fed back to the fluid solver to repeat the fixed-point iteration loop until the solid condition is satisfied. In general, this approach is more accurate when solid deflection is considerable. However, it is also more demanding in terms of computational time 
since both sets of equations need to be solved together and internal convergence should be reached during all iterations. The coupling between fluid and solid is enabled with stress and velocity constraints at their interface over all time steps. The force balance and no slip condition on the interface imply,

$$
\begin{gathered}
\sigma_{s} n=\sigma_{f} n \\
V_{s}=V_{f}
\end{gathered}
$$

where $n$ is the unit vector normal to the interface. The detailed coupling formulation can be found in reference [25].

\subsection{Computational domain and material properties}

The computational domain for the 2-D axisymmetric model is composed of fluid and solid regions, as illustrated in Fig. 1. The width of both domains is eight times the droplet radius $(R)$ and the heights of fluid and solid domains are $4 R$ and $10 R$, respectively. The gravitational force is exerted in the same direction as the droplet impingement. The fluid domain consists of air and water phases and the fluid properties at ambient conditions are utilized. The solid material is a titanium alloy, namely isotropic Ti-6Al-4V, which is widely utilized in gas turbine manufacturing.

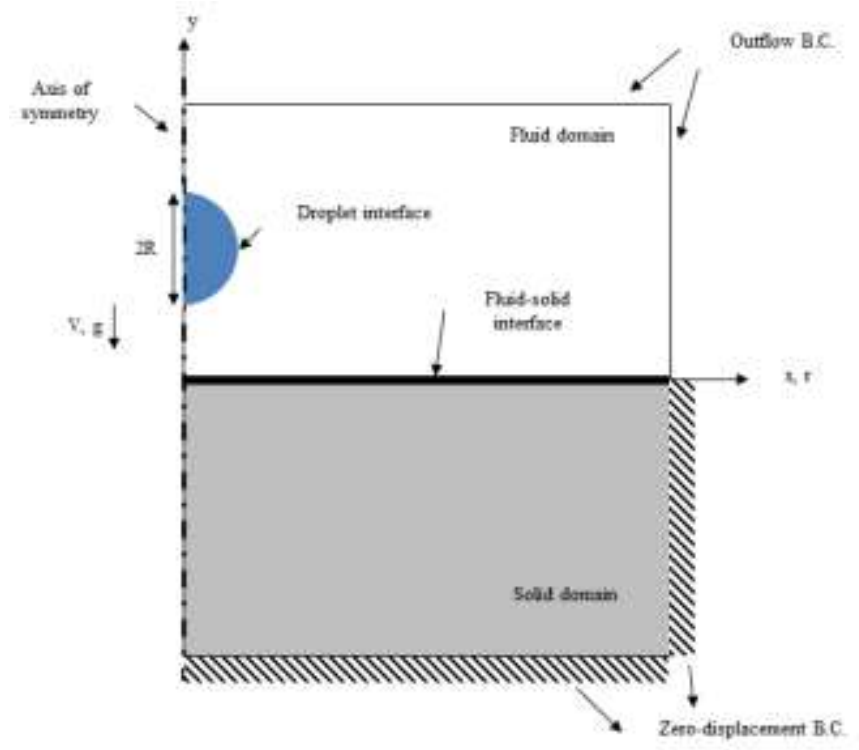

Fig. 1: Computational domain for 2-D axisymmetric FSI model.

\subsection{Numerical schemes \& Discretization}

The Pressure-Implicit with Splitting of Operators (PISO) method is used for pressure-velocity coupling in transient calculations. The integral forms of fluid equations are discretized over each control volume and solved for a fixed system of grids in a segregated manner. The solution is then obtained by using a time marching scheme based on the given initial condition. The discretization of the transport equations is second-order accurate in both space and time. The mesh is uniformly distributed in both domains with the same grid size. The time step during all the advective fluxes in transport equations is different from the one used in VOF calculations. The time step in VOF is adaptive and depends on the Courant-Friedrichs-Lewy (CFL) condition. CFL number is initially set to 0.1 to start the computations and the initial time step is determined based on the impact velocity and grid size accordingly.

OpenFoam, an open-source computational code, is used for programming the solver. The fluid and solid parts are solved with interFoam and stressedFoam, respectively. In OpenFoam, all the equations are solved in three dimensions and a 2-D axisymmetric mesh is simply a $5^{\circ}$ section of a full cylinder with flat sides and one cell in lateral direction, which results in a wedge. 


\section{Results and discussion}

The impact of incompressible droplets on rigid substrates is discussed first. To that end, only fluid domain is considered and the solid domain is not modeled, since it is assumed to be rigid. Afterwards, the results obtained from incompressible FSI model, composed of incompressible fluid model and elastic solid solver with two-way coupling, for impact velocities in incompressible regime are presented. The effect of substrate elasticity, as the basis for one-way or two-way coupling approach, is discussed. In presenting some of the results, the variables are nondimensionalized with respect to the following reference values: droplet radius $(R)$, impact velocity $\left(V_{0}\right)$ and water hammer pressure $\left(p_{w h}\right)$. As a convention, "*” denotes a dimensionless variable.

Fig. 2 (a) and (b) represent the dimensionless pressure along the radial and axial directions, respectively, for a droplet diameter of $500 \mu \mathrm{m}$ with an impingement velocity of $100 \mathrm{~m} / \mathrm{s}$. It should be mentioned that the water hammer pressure for this impact condition is equal to $143 \mathrm{MPa}$. As it is shown in Fig. 2, the dimensionless pressure at $\mathrm{t}^{*}=0$ is equal to 0.586 (83.8 $\mathrm{MPa})$ which is lower than the peak pressure, $\mathrm{p}^{*}=0.714(102.1 \mathrm{MPa})$, located at a radial distance of $\mathrm{r}^{*}=0.064(16 \mu \mathrm{m})$ from the axis. A close examination of the volume fraction at this time step, in Fig. 4, reveals that only $25 \%$ of the computational cells underneath the droplet are filled with liquid. This observation conveys the entrapment of an air layer with a height of $\mathrm{y}^{*}=0.016$ corresponding to $4 \mu \mathrm{m}$, right at the impact point. It should be mentioned that the grid size for this case is $1 \mu \mathrm{m}$, which has enough resolution to capture the air bubble formation.
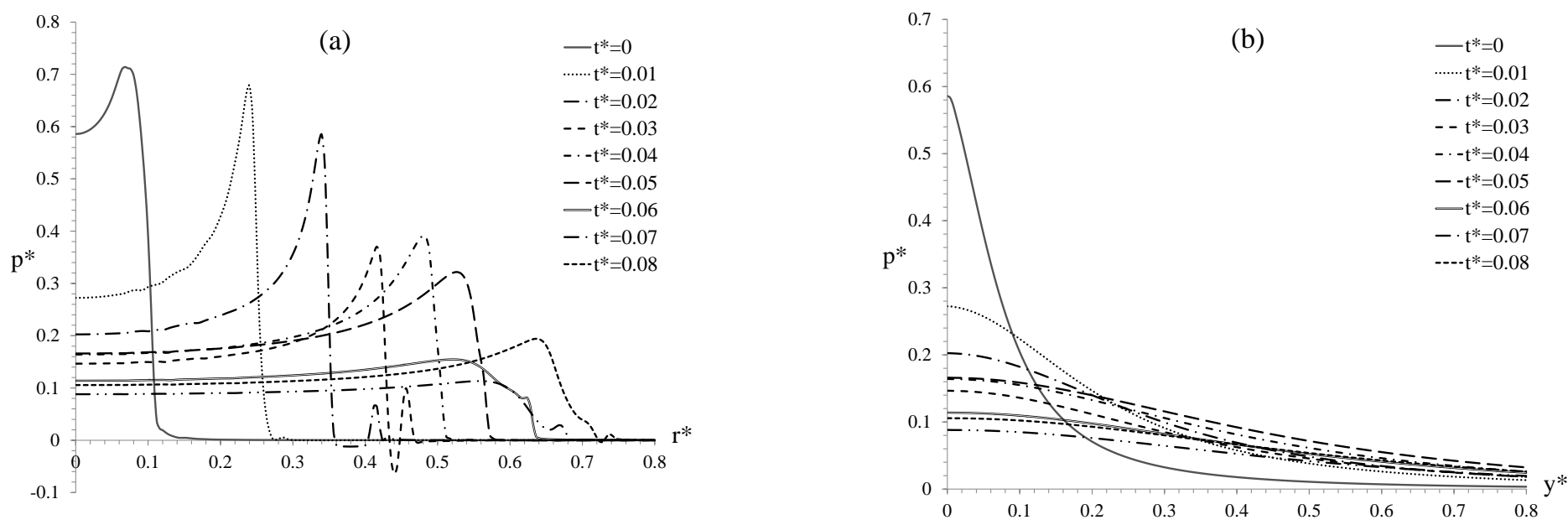

Fig. 2: Dimensionless pressure along (a) radial and (b) axial directions.

Fig. 3 (a) and (b) illustrate the variation of radial and vertical velocities, respectively, indicating that the radial and vertical velocity around the impact point is equal to zero, which confirms the formation of a stagnation region. In addition, as illustrated by the volume fraction graphs in Fig. 4, the air cushion formed upon the impact, which remains stationary at later time steps, has a height of $y^{*}=0.016(4 \mu \mathrm{m})$ and a radius of $r^{*}=0.06(15 \mu \mathrm{m})$. Furthermore, at the droplet periphery, the air escaping the impact point reaches a radial velocity of $\mathrm{V}_{\mathrm{r}}{ }^{*}=9.65(965 \mathrm{~m} / \mathrm{s})$ at $\mathrm{r}^{*}=0.144(36 \mu \mathrm{m})$, which is significantly higher that the initial impingement velocity of $100 \mathrm{~m} / \mathrm{s}$. It should be noted that there is no liquid at $\mathrm{r}^{*}=0.144$, confirming the presence of $100 \%$ air. 

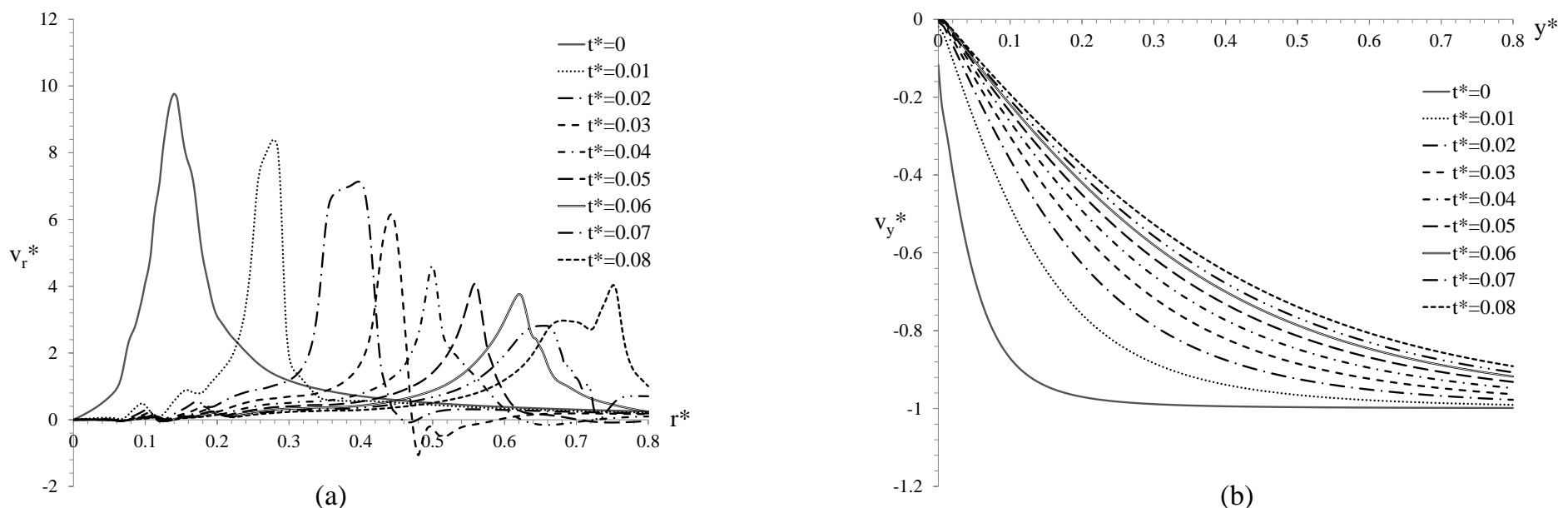

Fig. 3: Dimensionless (a) radial velocity and (b) axial velocity.

At $t^{*}=0.01$, the pressure is still high close to the droplet edge ( $\mathrm{p}^{*}=0.678$ at $\left.\mathrm{r}^{*}=0.24\right)$, but its magnitude is reduced by half at the center $\left(p^{*}=0.272\right)$. Moreover, the radial velocity is still high behind the droplet edge at $\mathrm{r}^{*}=0.276$ with a magnitude of $\mathrm{V}_{\mathrm{r}}{ }^{*}=8.35$. The vertical velocity remains zero at the impact point and its value decreases over time as the droplet continues its downward motion toward the surface, where its maximum magnitude occurs at liquid particles farther from the surface $\left(\mathrm{y}^{*}>0.8\right)$. At $\mathrm{t}^{*}=0.02$ and 0.03 , a negative pressure and radial velocity can be noticed in Fig. 2 (a) and Fig. 3 (a), respectively. This is due to the formation of a vortex. A rotational flow is generated at these time steps since the high velocity air behind the droplet that is spreading outward meets the still air in the surrounding. As a result, a reverse flow is formed with the air moving toward the droplet axis with a velocity of $\mathrm{V}_{\mathrm{r}}{ }^{*}=-1.06$ at $\mathrm{r}^{*}=0.48$, leading to the upward motion of the lateral jet at $t^{*}=0.04$. As the droplet continues to spread over the surface, the peak pressure and maximum radial velocity move outward from the center axis and their magnitudes degrade over time and eventually the pressure curve flattens.

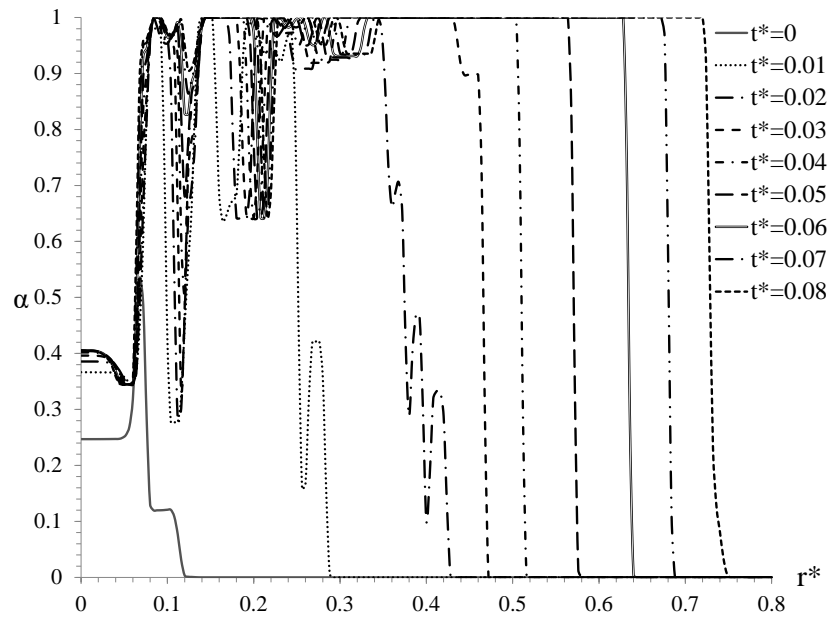

(a)

(b)

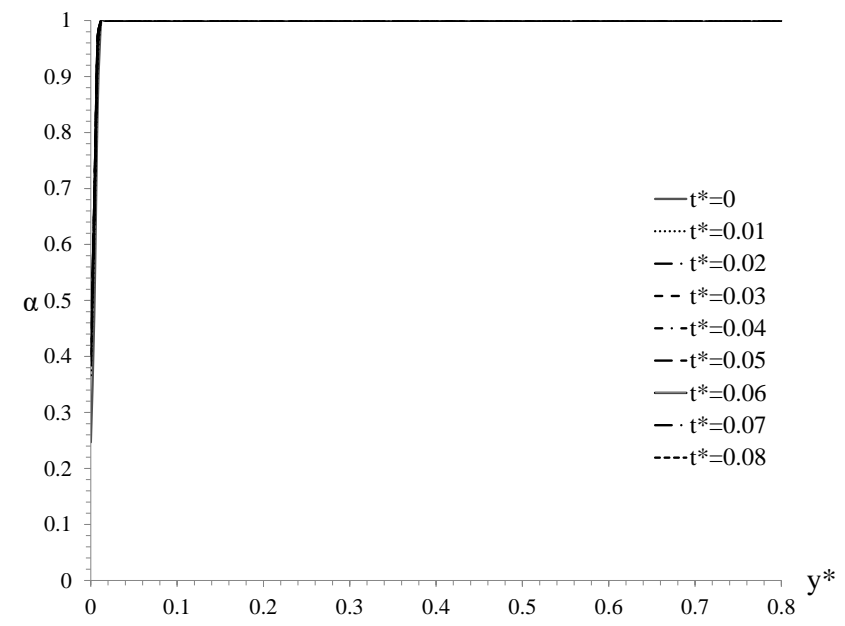

Fig. 4: Liquid volume fraction along (a) radial direction and (b) axial direction.

In order to investigate the effect of solid elasticity on the results, two cases are considered with the same impact conditions, one on a rigid substrate and the other one on an elastic plate. The maximum dimensionless pressure generated on the fluid-solid interface is compared against each other over dimensionless time, as displayed in Fig. 5 (a). It can be inferred from this comparison that the maximum pressure generated upon impact is slightly higher on a rigid solid, with less than $6 \%$ variation. Therefore, it would be a reasonable assumption that the solid elasticity has very little influence on the pressure build-up in the fluid and the consequent stress in the solid, since the two are closely connected. As a 
result, the fluid and solid equations can be decoupled and solved in a segregated manner to reduce the computational time significantly without losing much accuracy at incompressible impingement velocities.

The variation of the maximum pressure in the fluid and maximum stress in the solid along the interface (r-axis) are illustrated in Fig. 5 (b). The results are obtained from the incompressible fluid solver coupled with the elastic structural model using a two-way coupling scheme for the same impact scenario $\left(D=500 \mu \mathrm{m}, V_{0}=100 \mathrm{~m} / \mathrm{s}\right)$. This requires that both fluid and solid domains to be solved simultaneously and the variables on the fluid-solid interface to be exchanged at each time step until internal convergence is achieved. As it can be seen in the graph, the dimensionless pressure and stress display a similar behavior and reach a maximum value of 0.75 and 0.62 , respectively.

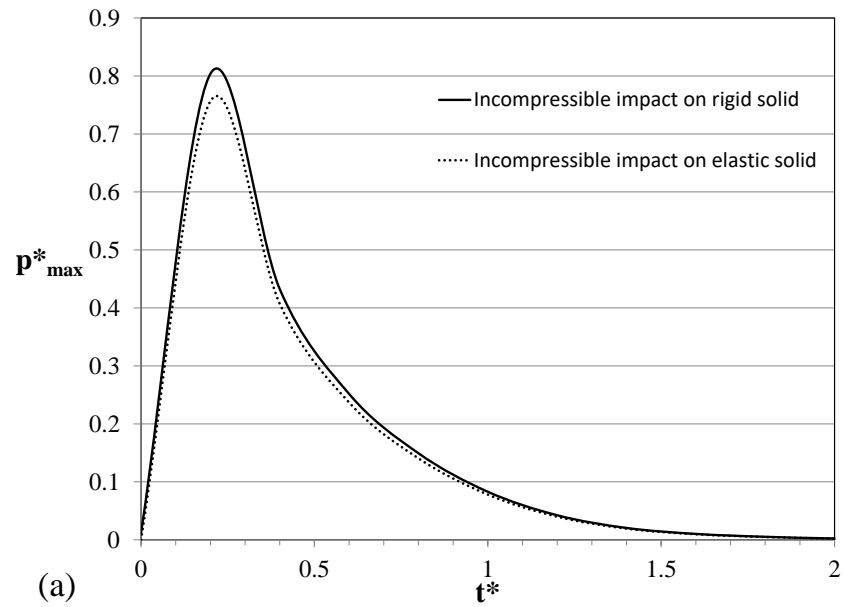

Fig. 5: (a) Effect of solid elasticity on maximum pressure, (b) maximum pressure and stress along the interface vs. time.

\section{Conclusion}

Fluid-solid interaction modeling was performed for single droplet impingements at relatively high velocities utilizing one-way and two-way coupling approaches. The results confirmed the formation of a layer of air underneath the droplet between the liquid and solid surface. Due to the air entrapment, the lateral liquid jet can reach velocities as high as ten times the impact velocity. In addition, the high-pressure region, formed close to the droplet axis at early stages, moves radially outward toward the droplet edge during the spreading of the droplet on the solid surface. Furthermore, it was illustrated that the solid elasticity leads to a lower pressure build-up on the surface. The comparison between the elastic and rigid substrates revealed that the solid elasticity does not have a significant influence on the maximum pressure history generated by the impact. In this regard, the fluid and solid equations can be solved in a segregated manner using a one-way coupling approach for the tested case, i.e. $100 \mathrm{~m} / \mathrm{s}$ impingement speed.

\section{Acknowledgements}

This work was funded by Consortium de Recherche et d'Innovation en Aérospatiale au Québec (CRIAQ), Natural Sciences and Engineering Research Council of Canada (NSERC), Le Fonds Québécois de la Recherche sur la Nature et les Technologies (FQRNT), Nano-Quebec, Hydro Quebec and Siemens Canada (formerly Rolls-Royce Canada).

\section{References}

[1] E. Honegger, "Test on Erosion caused by Jets," vol. 14, pp. 95-104, 1927.

[2] H. W. Bargmann, "The Mechanics of Erosion by Liquid and Solid Impact," vol. 29, no. 1415, pp. 1685-1698, 1992.

[3] G. S. Springer, "Erosion by liquid impact," 1976.

[4] J. Fukai, Y. Shiiba, T. Yamamoto, O. Miyatake and D. Pouli, "Wetting effects on the spreading of a liquid droplet colliding with a flat surface: experiment and modeling," vol. 7, no. 2, pp. 236-247, 1995.

[5] Chandra S. and C. T. Avedisian, "On the Collision of a Droplet with a Solid Surface," vol. 432, no. 1884, 1991. 
[6] M. Pasandideh-Fard, Y. M. Qiao, S. Chandra and J. Mostaghimi, "Capillary effects during droplet impact on a solid surface," vol. 8, no. 3, 1996.

[7] I. V. Roisman, R. Rioboo and C. Tropea, "Normal impact of a liquid drop on a dry surface: model for spreading and receding," vol. 458, no. 2022, 2002.

[8] V. Mehdi-Nejad, J. Mostaghimi and S. Chandra, "Air bubble entrapment under an impacting droplet," vol. 15, no. 1, pp. 173-183, 2003.

[9] N. Z. Mehdizadeh, S. Chandra and J. Mostaghimi, "Formation of fingers around the edges of a drop hitting a metal plate with high velocity," vol. 510, pp. 353-373, 2004.

[10] T. D. Blake and J. De Conick, "The influence of solid-liquid interactions on dynamic wetting," vol. 96, no. 1-3, pp. 21-36, 200.

[11] S. S. Cook, "Erosion by Water Hammer,” vol. 119, pp. 481-8, 1928.

[12] O. G. Engel, "Waterdrop Collisions with Solid Surface," vol. 54, 1955.

[13] R. M. Blowers, “On the Response of an Elastic Solid to Droplet Impact,” vol. 5, pp. 167-193, 1969.

[14] W. F. Adler and D. J. Mihora, “Analysis of Water Drop Impacts on Layered Window Constructions,” pp. 264-274, 1994.

[15] K. K. Haller, Y. Ventikos, D. Poulikakos and P. Monkewitz, "Computational Study of High-speed Liquid Droplet Impact," vol. 92, pp. 2821-8, 2002.

[16] Y. C. Huang, F. G. Hammitt and W. J. Yang, "Hydrodynamic phenomena during high-speed collision between liquid droplet and rigid plane," vol. 95, pp. 276-294, 1973.

[17] N. Li, Q. Zhou, X. Chen, T. Xu, S. Hui and D. Zhang, "Liquid drop impact on solid surface with application to water drop erosion on turbine blades, Part I: Nonlinear wave model and solution of one-dimensional impact," vol. 50, pp. 1526-1542, 2008.

[18] Q. Zhou, N. Li, X. Chen, T. Xu, S. Hui and D. Zhang, "Liquid drop impact on solid surface with application to water drop erosion on turbine blades, Part II: Axisymmetric solution and erosion analysis," vol. 50, pp. 1543-1558, 2008.

[19] M. Marzbali, "Numerical Analysis of High-speed Droplet Impingement on Elastic and Rigid Substrates," Concordia University, 2017.

[20] M. Marzbali, A. Dolatabadi and P. Jedrzejowski, "Fluid-Solid Interaction Modeling of Compressible Droplet Impact onto Elastic Substrates," in 21st AIAA Computational Fluid Dynamics Conference, San Diego, 2013.

[21] R. Li, H. Ninokata and M. Mori, "A numerical study of impact force caused by liquid droplet impingement onto a rigid wall," vol. 53, pp. 881-885, 2011.

[22] J. U. Brackbill, D. B. Kothe and C. Zemach, “A continuum method for modelling surface tension,” vol. 100, no. 2, p. 335-354, 1992.

[23] C. Hirt and B. Nichols , "Volume of Fluid (VOF) Method for the Dynamics of Free Boundaries," vol. 39, no. 1, pp. 201-225, 1981.

[24] D. L. Youngs, “Time-Dependent Multi-Material Flow with Large Fluid Distortion," Numerical Methods for Fluid Dynamics, 1982.

[25] S. Turek and J. Hron, "Proposal for Numerical Benchmarking of Fluid-Structure Interaction between an Elastic Object and Laminar Incompressible Flow,” in Fluid-Structure Interaction, Springer, 2006. 